\title{
Driving Risk Detection Model of Deceleration Zone in Expressway Based on Generalized Regression Neural Network
}

\author{
Weiwei Qi $\mathbb{D}^{1,2}$ Zhexuan Wang, ${ }^{1,}$ Ruru Tang, and Linhong Wang $\mathbb{D}^{4}$ \\ ${ }^{1}$ School of Civil Engineering and Transportation, South China University of Technology, Guangzhou 510641, China \\ ${ }^{2}$ School of Civil and Environmental Engineering, University of Technology Sydney, Sydney, NSW 2007, Australia \\ ${ }^{3}$ School of Transportation Science and Engineering, Harbin Institute of Technology, Harbin 150090, China \\ ${ }^{4}$ School of Transportation, Jilin University, Changchun 130022, China
}

Correspondence should be addressed to Linhong Wang; wanghonglin0520@126.com

Received 20 July 2018; Accepted 24 September 2018; Published 10 October 2018

Guest Editor: David Z. W. Wang

Copyright (c) 2018 Weiwei Qi et al. This is an open access article distributed under the Creative Commons Attribution License, which permits unrestricted use, distribution, and reproduction in any medium, provided the original work is properly cited.

\begin{abstract}
Drivers' mistakes may cause some traffic accidents, and such accidents can be avoided if prompt advice could be given to drivers. So, how to detect driving risk is the key factor. Firstly, the selected parameters of vehicle movement are reaction time, acceleration, initial speed, final speed, and velocity difference. The ANOVA results show that the velocity difference is not significant in different driving states, and the other four parameters can be used as input variables of neural network models in deceleration zone of expressway, which have fifteen different combinations. Then, the detection model results indicate that the prediction accuracy rate of testing set is up to $86.4 \%$. An interesting finding is that the number of input variables is positively correlated with the prediction accuracy rate. By applying the method, the dangerous state of vehicles could be released through mobile internet as well as drivers' start of risky behaviors, such as fatigue driving, drunk driving, speeding driving, and distracted driving. Numerical analyses have been conducted to determine the conditions required for implementing this detection method. Furthermore, the empirical results of the present study have important implications for the reduction of crashes.
\end{abstract}

\section{Introduction}

The road traffic system consists of drivers, roads, vehicles, and environment. Traffic safety is an important part in the field of social management, and $90 \%$ of traffic accidents are caused by drivers' risky behavior $[1,2]$. There are four typical risky driving behaviors: fatigue driving, drunk driving, speeding driving, and distracted driving. Human, vehicle, road, and environmental risk factors are connected with fatigue-related traffic accidents, and fatigue driving is a major cause of traffic accidents [3]. Drunk driving is one of the major behavioral issues connected with problematic alcohol consumption [4]. Speed is one of the most important factors in traffic safety as higher speeds are linked to increased crash risk and higher injury severities [5]. The young adults are easily at the highest risk of engaging in distracted driving [6]. In this paper, the state of driving risk is the behaviors during deceleration zone, which can easily cause adverse consequences.
The process of driving behavior will be presented in specific vehicle movement. The parameters of vehicle movement are generally vehicle speed, acceleration, and vehicle trajectory [7]. So, the driving risk can be identified by observing parameters of vehicle movement [8]. Many measures have been proposed to monitor the driving risk, but they mainly focus on the determination of the driver's physiological indicators [9-11]. Neural network is an important method to detect driving risk [12]. With the fast development and adoption of vehicle-to-infrastructure (V2I) facilities, it is very convenient to detect driving risk, and the accuracy of the detection model is a key factor [13-15]. So, a neural network model of high accuracy will be established to identify driving risk in this paper.

Speed is one of the most important factors in traffic safety, and on-ramps and off-ramps in expressway are typical sections [16]. The work zone in expressways has higher rearend crash risk than that in arterials [17]. There is strong 
TABLE 1: Set of characterization indicators for driving risk.

\begin{tabular}{|c|c|c|c|}
\hline Variable name & Unit & Indicators & Description \\
\hline$t_{\mathrm{R}}$ & s & Reaction time & $\begin{array}{l}\text { It characterizes the zone time } \\
\text { between the drivers' reading the } \\
\text { speed limit sign and vehicles } \\
\text { slowing down. }\end{array}$ \\
\hline$v_{\mathrm{I}}$ & $\mathrm{m} / \mathrm{s}$ & Initial speed & $\begin{array}{l}\text { It characterizes the speed at } \\
\text { which the driver finds the speed } \\
\text { limit sign. }\end{array}$ \\
\hline$v_{\mathrm{F}}$ & $\mathrm{m} / \mathrm{s}$ & Final speed & $\begin{array}{l}\text { It characterizes the speed which } \\
\text { the driver can keep after the } \\
\text { deceleration is completed. }\end{array}$ \\
\hline$\Delta v$ & $\mathrm{~m} / \mathrm{s}$ & Velocity difference & $\begin{array}{l}\text { It characterizes the change in } \\
\text { speed for the driver after seeing } \\
\text { the speed limit sign. }\end{array}$ \\
\hline$a$ & $\mathrm{~m} / \mathrm{s}^{2}$ & Acceleration & $\begin{array}{c}\text { It characterizes the rate of the } \\
\text { speed change during } \\
\text { deceleration. }\end{array}$ \\
\hline
\end{tabular}

relationship between speed compliance and the frequency and severity of traffic accidents [18]. Vehicle speed changing and driver reaction time can be measured accurately in deceleration zone of expressway $[19,20]$. So, five typical parameters are selected to represent the vehicle speed changing and driver reaction time. Besides, a generalized regression neural network (GRNN) is used as a correlation method of the five typical parameters [21].

The study of driving risk detection is carried out based on GRNN model in deceleration zone of expressway, which aims to reduce the incidence of accidents and provide security for drivers' safety.

\section{Influencing Factors Analysis for Driving Risk}

According to the relevant research results, there are three main factors to affect the driver's driving ability [22], which are as follows:

(1) Fatigue causes the driver's body function to be unbalanced

(2) Distracted driving makes it difficult for drivers to concentrate their attention, due to interference from external factors

(3) There is temporary injury of the driver, such as a decrease in the actual working ability of the driver, which is due to side effects of drugs and alcohol

The state of vehicle in the deceleration zone is a specific expression of the situation in which the driver manipulates the vehicle, and it can determine whether the driver is in a dangerous condition. An important feature of drivers in a dangerous situation is an increase in reaction time of the operating vehicle, which is manifested in the running state of the vehicle. So the reaction time of deceleration is longer when traveling through the deceleration zone. The related studies have shown that drivers who are in dangerous driving conditions often respond to sudden speed brakes to reduce the speed of the vehicle. Therefore, excessive acceleration is a poor condition for driving. In addition, when the vehicle is traveling through the deceleration zone, if the speed is too large and the speed is not lowered than the prescribed speed, it is also a dangerous driving state [23]. So, it is useful to detect drivers' state via parameters of vehicle movement.

In order to establish the detection model for driving risk, the five indicators are initially selected as characterization indicators of the danger driving assessment, which are reaction time, acceleration, initial speed, final speed, and velocity difference. The details are given in Table 1. According to the characteristics of the deceleration zone, we designed the detection process of dangerous driving state, which is as showed in Figure 1.

\section{Experimental Testing and Data Optimization}

The goal of this experiment is to collect data of drivers and vehicles in the deceleration zone, such as acceleration, reaction time, initial speed, final speed, and velocity difference. It is the data foundation for constructing the detection model for driving risk.

3.1. Experimental Implementation Process. Based on the experimental purpose, we have developed a comprehensive data acquisition program via simulated driving experiments. We chose the scene of expressway deceleration zone in the simulated driving platform as the typical section, and the simulated driving platform contains multiple sensors for collecting behavior and vehicle signals, which has been applied to various driving behavior experiments [24].

There are four driving states of "normal driving," "speeding driving," "distracted driving," and "fatigue driving." Each driver continues to be tested for about 30 minutes under the four driving conditions. Experimental equipment collects drivers' physiological information, vehicle operating parameters, and driving environment information in real time. Drivers control the simulated driving platform to complete 


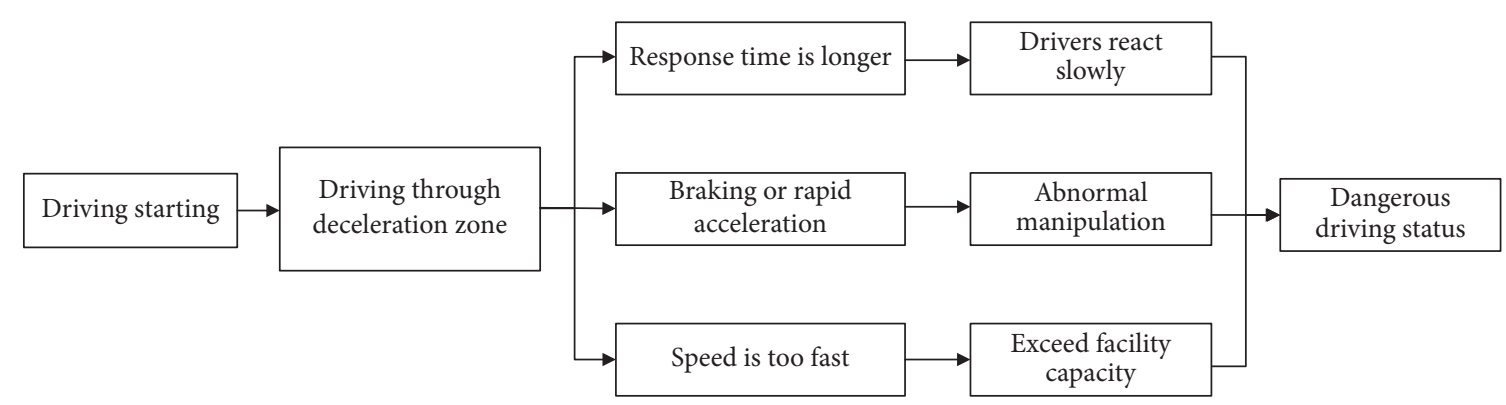

FIGURE 1: Detection process of dangerous driving state.

TABLE 2: Parts of the original sample data.

\begin{tabular}{lccccc}
\hline Status & Acceleration & Reaction time & Initial speed & Final speed & Velocity difference \\
\hline Normal & -6.38 & 0.48 & 106 & 50 & 56 \\
Normal & -5.66 & 0.24 & 104 & 51 & 53 \\
Normal & -6.14 & 0.32 & 98 & 70 & 28 \\
Danger & -2.41 & 0.88 & 115 & 80 & 35 \\
Danger & -2.16 & 0.84 & 115 & 95 & 20 \\
Danger & -2.28 & 1.08 & 130 & 110 & 20 \\
$\ldots$ & $\ldots$ & $\ldots$ & $\ldots$ & $\ldots$ & $\ldots$ \\
\hline
\end{tabular}

the natural operation according to the requirements of the experimental operation process, and the multiple sensors collect five required indicators, namely, the initial speed $v_{\mathrm{I}}$, the final speed $v_{\mathrm{F}}$, the velocity difference $\Delta v$, the reaction time $t_{\mathrm{R}}$, and the acceleration $a$.

When the experiment is completed, the data of each driver's personality, vehicle running status, and driving environment are summarized, and the driving experimental database in the expressway deceleration zone is established to complete the detection model.

3.2. Extraction for Effective Data. According to the experimental scheme, we need to obtain the information of five raw parameters via the multiple sensors, and the parameters are the initial speed $v_{\mathrm{I}}$, the corresponding time $t_{1}$ when the drivers see the speed limit sign, the time $t_{2}$ when driver's action starts to decelerate via seeing the speed limit sign, the time $t_{3}$ when the drivers decelerate to a certain speed and are about to move at a constant speed, and the final speed $v_{\mathrm{F}}$ at time $t_{3}$. After obtaining the above sample data, the reaction time $t_{\mathrm{R}}$, the velocity difference $\Delta v$, and the acceleration $a$ can be calculated according to formulas (1), (2), and (3). After the above processing, we can get the five required indicators, namely, the initial speed $v_{\mathrm{I}}$, the final speed $v_{\mathrm{F}}$, the velocity difference $\Delta v$, the reaction time $t_{\mathrm{R}}$, and the acceleration $a$.

$$
\begin{gathered}
t_{\mathrm{R}}=t_{2}-t_{1} \\
\Delta v=v_{\mathrm{F}}-v_{\mathrm{I}} \\
a=\frac{v_{F}-v_{I}}{t_{3}-t_{2}}
\end{gathered}
$$

In this simulation experiment, each driver has to perform three normal driving processes and three dangerous driving processes. The dangerous driving process is divided into speeding driving, distracted driving, and fatigue driving. For normal driving, we stipulate that the driving speed cannot exceed the speed limit value of the relevant section in the expressway. Since the maximum speed of the expressway in China is $120 \mathrm{~km} / \mathrm{h}$, the parts of data whose speed is larger than $120 \mathrm{~km} / \mathrm{h}$ have been excluded. For dangerous driving, due to the simulation characteristics and the errors in the experimental operation, we also have eliminated the invalid data. The style and composition of data are shown in Table 2.

In this experiment, we have obtained more than 60 hours of data from 31 drivers, and the uniform sampling method is used to extract 332 sets of valid data in the normal and dangerous state [25]. Specifically, there are 266 samples in the training set, 133 samples of which are in the normal state and dangerous state, respectively; there are 66 samples in the testing set, 33 samples of which are in the normal state and dangerous state, respectively.

3.3. Screening for Optimal Characterization. Table 2 is the raw data set based on multiple sensors, and each set of data contains five indicators of acceleration, reaction time, initial speed, final speed, and velocity difference. In the next step, the one-way ANOVA is used to test whether the five indicators have significant differences between normal and dangerous states, and the tested indicators are selected as the best indicators of driving risk [26]. The test results are given in Table 3.

The analysis results in Table 3 show that there are significant differences $(\mathrm{P}<0.05)$ in the initial speed, final speed, reaction time, and acceleration between normal and dangerous states. This is to say, the above four indicators can be used as the ideal indicators of driving risk. However, the 
TABLE 3: Variance analysis results of various indicators.

\begin{tabular}{lccccc}
\hline Index & df & MS & F & P value & F-crit \\
\hline Initial speed & 1 & 11462.93 & 107.65 & $5.54 \mathrm{E}-22$ & 3.87 \\
Final speed & 1 & 14432.71 & 102.30 & $4.28 \mathrm{E}-21$ & 3.87 \\
Velocity difference & 1 & 170.85 & 1.10 & 0.2944 & 3.87 \\
Reaction time & 1 & 42.68 & 6.87 & 0.0092 & 3.87 \\
Acceleration & 1 & 3.20 & 61.77 & $5.68 \mathrm{E}-14$ & 3.87 \\
\hline
\end{tabular}

Input layer Pattern layer Summation layer Output layer

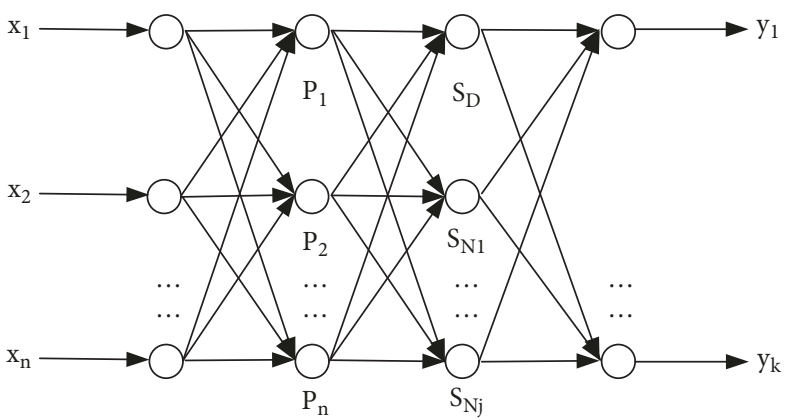

FIGURE 2: Classic structure for generalized regression neural network.

velocity difference did not pass the ANOVA testing. So, the four indicators of acceleration, reaction time, initial speed, and final speed will be used as indicators for the detection mode recognition of driving risk.

\section{Detection Model of Driving Risk Based on GRNN}

4.1. Structure for Classic GRNN. In many nonlinear models that solve the problem of supervised pattern recognition, the artificial neural network models are widely used due to their excellent adaptive ability. Besides, in actual modeling and computation, the GRNN exhibits higher prediction accuracy and faster computational speed than other nonlinear models. So, the GRNN model has been selected to solve this pattern recognition problem. GRNN is a feed-forward neural network model based on nonlinear regression theory, which is composed of four layers in the structure, which are input layer, pattern layer, summation layer, and output layer [27]. The classic structure of GRNN is shown in Figure 2.

The input matrix is $\mathrm{X}=\left[x_{1}, x_{2}, \ldots, x_{n}\right]^{T}$, and the output matrix is $\mathrm{Y}=\left[y_{1}, y_{2}, \ldots, y_{k}\right]^{T}$. The number of input layer neurons is equal to the dimension of the input matrix in the training sample. The input layer only sends the sample variables to the pattern layer and does not participate in the real operation. The number of pattern layer neurons is equal to the number of training set samples, and the layer neuron transfer function is

$$
P_{i}=e^{\left[-\left(X-X_{i}\right)^{T}\left(X-X_{i}\right) / 2 \sigma^{2}\right]} \quad i=1,2, \ldots, n,
$$

The summation layer uses two types of neurons for summation. The first type computes the sum of the output lines of all pattern layer neurons. The pattern layer and each neuron have a connection weight of 1 , and the first type summation function is

$$
S_{D}=\sum_{i=1}^{n} P_{i},
$$

The second type weights the neurons of all pattern layers, and the connection weight between the pattern layer and each neuron is taken as the output matrix of training set, and the second type summation function is

$$
S_{N j}=\sum_{i=1}^{n}\left(y_{i j} P_{i}\right), \quad j=1,2, \ldots, k,
$$

Each neuron in the output layer is associated with the two type results of the summation layer. The output function is

$$
y_{j}=\frac{S_{N j}}{S_{D}} \quad j=1,2, \ldots, k,
$$

The GRNN can achieve good nonlinear mapping ability and learning speed. Its weight and threshold are given in the training sample in one step. It does not have to be iterated, and the calculation amount is small.

4.2. Algorithm Process of GRNN. The GRNN has been used to construct the detection model of driving risk in deceleration zone of expressway, and the algorithm process is as follows [28].

4.2.1. Generating Training Sets and Testing Sets. To avoid generality, we use a random method to generate training sets and testing sets. As mentioned above, the experimental data includes the categories of driving status (normal driving 
TABLE 4: Sample data for training sets and testing sets.

\begin{tabular}{lcccc}
\hline Status & Acceleration & Reaction time & Initial speed & Final speed \\
\hline Normal & -6.38 & 0.48 & 106 & 50 \\
Normal & -5.66 & 0.24 & 104 & 51 \\
Normal & -6.14 & 0.32 & 98 & 70 \\
Danger & -2.41 & 0.88 & 115 & 80 \\
Danger & -2.16 & 0.84 & 115 & 95 \\
Danger & -2.28 & 1.08 & 130 & 110 \\
$\ldots$ & $\ldots$ & $\ldots$ & $\ldots$ & $\ldots$ \\
\hline
\end{tabular}

TABLE 5: Input variable combinations corresponding to the 15 models.

\begin{tabular}{|c|c|c|c|c|}
\hline \multirow{2}{*}{ Model number } & \multicolumn{4}{|c|}{ Input variable } \\
\hline & Acceleration & Reaction time & Initial speed & Final speed \\
\hline 1 & $\bigcirc$ & & & \\
\hline 2 & 0 & $\bigcirc$ & & \\
\hline 3 & 0 & 0 & 0 & \\
\hline 4 & 0 & 0 & 0 & 0 \\
\hline 5 & & $\mathrm{O}$ & & \\
\hline 6 & & $\bigcirc$ & 0 & \\
\hline 7 & & 0 & 0 & 0 \\
\hline 8 & & & 0 & \\
\hline 9 & & & 0 & 0 \\
\hline 10 & & & & 0 \\
\hline 11 & 0 & & 0 & \\
\hline 12 & $\bigcirc$ & & & 0 \\
\hline 13 & $\bigcirc$ & $\bigcirc$ & & $\bigcirc$ \\
\hline 14 & 0 & & 0 & 0 \\
\hline 15 & & 0 & & 0 \\
\hline
\end{tabular}

and dangerous driving) and four indicators (acceleration, reaction time, initial speed, and final speed). Some sample data are shown in Table 4. Specifically, there are 266 samples in the training set and 66 samples in the testing set.

4.2.2. Creating GRNN Model Group for Driving Risk. We use the toolbox function "newgrnn" of MATLAB neural network to create the GRNN models, and a total combination of the four variables has been established. It can be divided into 15 groups, and each model is constructed separately. For example, the variable of input layer in model 1 is the initial speed, and the variables of input layer in model 2 are the initial speed and final speed. The combinations for input variables are shown in Table 5 corresponding to the 15 GRNN models.

4.2.3. Simulation Testing. After the GRNN model for driving risk is established, the input matrix of the testing set can be taken into the model, and the output of the model is the corresponding prediction result.

4.2.4. Performance Evaluation. By calculating the error between the prediction category and the actual category of the testing set, the generalization ability of the GRNN model on driving risk can be evaluated. At the same time, the MATLAB function "cputime" can be used to calculate the running time of the program, which is to measure the running speed and performance of the program.

4.3. Discussion of Results. Since the training set and the testing set are randomly generated, the results of every calculation are slightly different. The consequences of the certain program operation have been randomly selected. So, the prediction accuracy, running time, and optimal model testing results of the model group are, respectively, shown in Figures 3, 4, and 5. After analysis, the GRNN model group of the driving risk detection in deceleration zone can clearly give the following results:

(1) It can be seen from Figure 3 that the GRNN models have good generalization ability, and the best prediction accuracy rate of testing set is up to $86.4 \%$ (2) As can be seen from Figure 3, the numbers of GRNN model established by inputting each variable alone are model 1 , model 5 , model 8 , and model 10 (acceleration, reaction time, initial speed, and final speed). Among them, the GRNN model established by acceleration alone has the worst performance, and the 


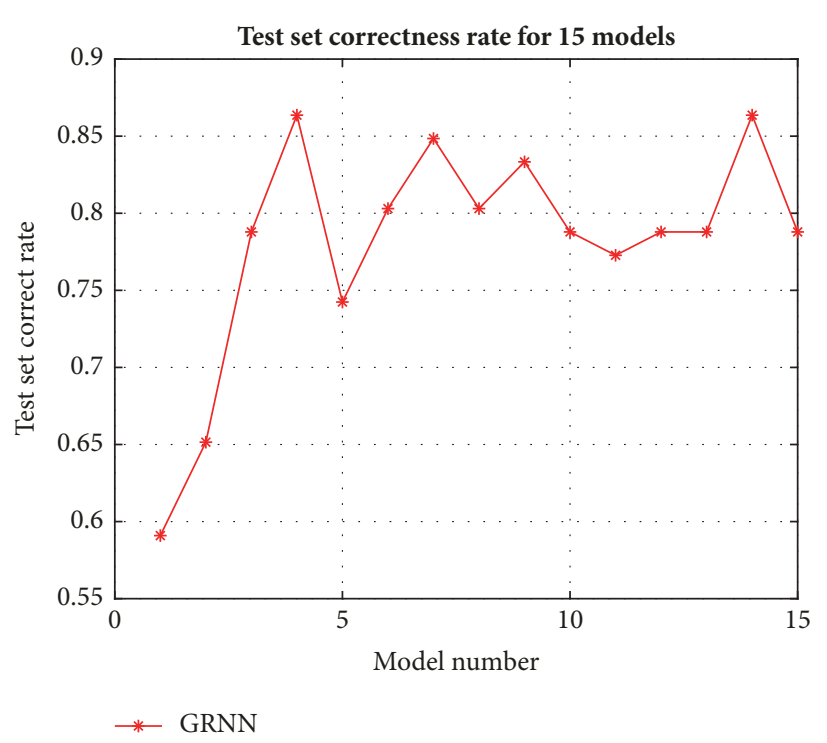

Figure 3: Prediction accuracy of the fifteen models.

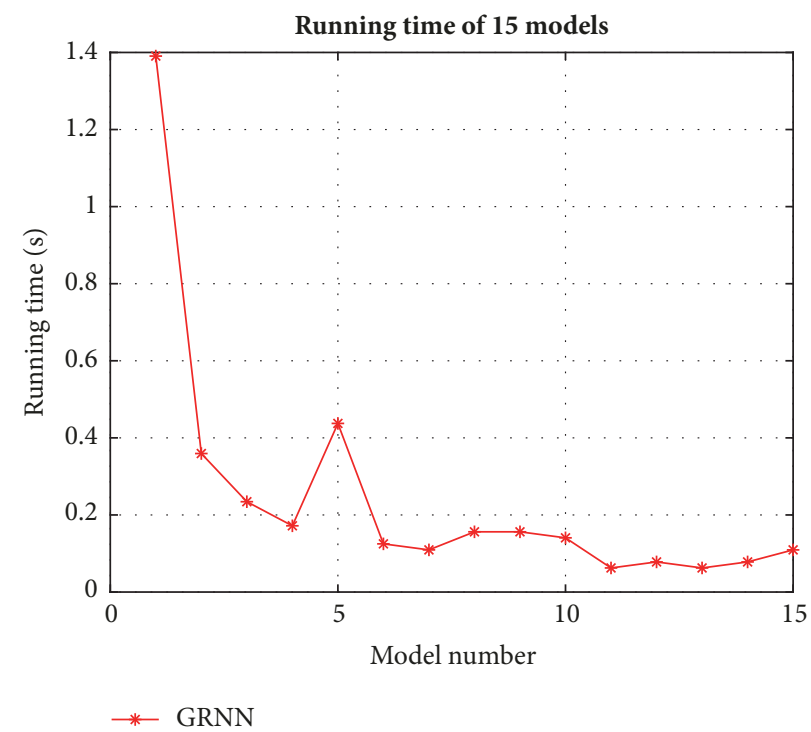

FIgURE 4: Running time of the fifteen models.

correct rate is just $59 \%$. The GRNN models with separate input of reaction time, initial speed, and final speed have better performance, and the correct rate is, respectively, $74 \%$, $80 \%$, and $79 \%$. This illustrates that the correlation between acceleration and vehicle motion is small, but the correlation between initial speed, final speed, reaction time, and vehicle motion is large

(3) In Figure 3, the input variable of model 1 is acceleration, the input variables of model 2 are acceleration and reaction time, the input variables of model 3 are acceleration, reaction time, and initial speed, and the input variables of model 4 are acceleration, reaction time, initial speed, and final speed. It can be seen from Figure 3 that the prediction accuracy is gradually improved from model 1 to model 4, and the same rules are applied to models 5-7 and models 8-9, which indicates that multiple factors contribute to the recognition of the driving state. The number of input variables is positively correlated with the prediction accuracy rate

(4) It can be seen from Figure 4 that the average running time of the 15 models is about $0.2 \mathrm{~s}$, which is much faster than the BP neural network algorithm

(5) The prediction result of testing set drawn in Figure 5 is the corresponding optimal driving risk detection for model 4 , and it is a typical model in which four parameters (acceleration, reaction time, initial speed, and final speed) are involved in the operation. The prediction accuracy of model 4 is $86.4 \%$, which is higher than the other models 


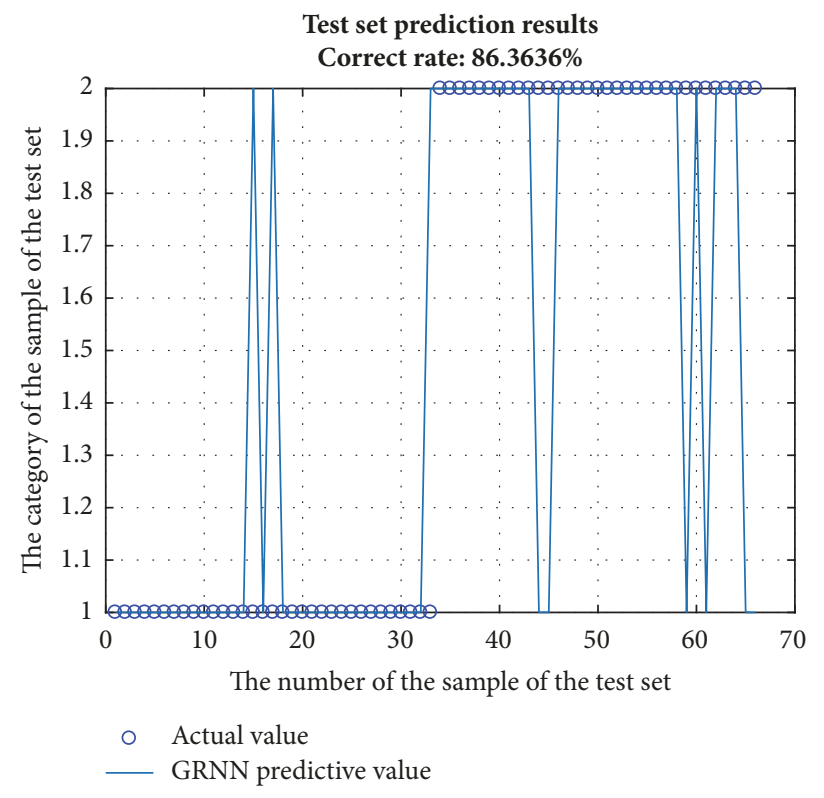

Figure 5: Prediction results of model 4.

\section{Conclusion}

One of the negatively influential issues regarding social life is a traffic accident. It has negative impacts on people's lives and well-being due to increasing their travel pressure. It has to be mentioned that it would not only threaten individuals' health, but also disturb the management system because of economic loss.

The study aims to develop driving risk detection model of deceleration zone in expressway. In this research, it is considered to increase prediction accuracy for the GRNN model. The four parameters have been selected via the ANOVA analysis. So, it is clear that number of input variables is positively correlated with the prediction accuracy rate. For this purpose, fifteen models are proposed to choose the best prediction model. Therefore, the risk of driving would be decreased remarkably if the GRNN model is used to detect vehicle movement.

In the future work, there are two aspects to improve. Firstly, more parameters should be collected and input to the GRNN model, in order to heighten the prediction accuracy rate. Then, data from actual traffic conditions are available to validate the simulated data.

\section{Data Availability}

The "variance analysis results of various indicators" data used to support the findings of this study are included within the article. The "sample data for training sets and testing sets" data used to support the findings of this study are currently under embargo while the research findings are commercialized. Requests for data, 12 months after publication of this article, will be considered by the corresponding author.

\section{Conflicts of Interest}

The authors declare that they have no conflicts of interest.

\section{Acknowledgments}

The study is supported by the National Natural Science Foundation of China (NO. 71701070), the Natural Science Foundation of Guangdong Province (NO. 2016A030310427), the Science and Technology Project of Guangzhou City (NO. 201804010466), and the China Scholarship Council.

\section{References}

[1] X. Qu, Q. Meng, and Z. Liu, "Estimation of number of fatalities caused by toxic gases due to fire in road tunnels," Accident Analysis \& Prevention, vol. 50, pp. 616-621, 2013.

[2] C. Wang and N. Stamatiadis, "Sensitivity analysis on new simulation-based conflict metrics," Safety Science, vol. 82, pp. 399-409, 2016.

[3] G. Zhang, K. K. W. Yau, X. Zhang, and Y. Li, “Traffic accidents involving fatigue driving and their extent of casualties," Accident Analysis \& Prevention, vol. 87, pp. 34-42, 2016.

[4] I. Taymur, E. Budak, V. Duyan, B. B. Kanat, and S. Önen, "Examination of personality traits and social problem-solving skills of individuals whose driving licenses have been confiscated due to drunk driving," Traffic Injury Prevention, vol. 18, no. 1, pp. 3-8, 2017.

[5] C. D. Fitzpatrick, S. Rakasi, and M. A. Knodler, "An investigation of the speeding-related crash designation through crash narrative reviews sampled via logistic regression," Accident Analysis \& Prevention, vol. 98, pp. 57-63, 2017.

[6] K. A. Braitman and A. L. Braitman, "Patterns of distracted driving behaviors among young adult drivers: Exploring relationships with personality variables," Transportation Research 
Part F: Traffic Psychology and Behaviour, vol. 46, pp. 169-176, 2017.

[7] Z. Liu, W. Yi, S. Wang, and J. Chen, "On the Uniqueness of User Equilibrium Flow with Speed Limit," Networks and Spatial Economics, vol. 17, no. 3, pp. 763-775, 2017.

[8] C. Wang, C. Xu, J. Xia, Z. Qian, and L. Lu, "A combined use of microscopic traffic simulation and extreme value methods for traffic safety evaluation," Transportation Research Part C: Emerging Technologies, vol. 90, pp. 281-291, 2018.

[9] R. Sayed and A. Eskandarian, "Unobtrusive drowsiness detection by neural network learning of driver steering," Proceedings of the Institution of Mechanical Engineers, Part D: Journal of Automobile Engineering, vol. 215, no. 9, pp. 969-975, 2001.

[10] M. V. M. Yeo, X. P. Li, K. Q. Shen, and E. P. V. Wilder-Smith, "Can SVM be used for automatic EEG detection of drowsiness during car driving?” Safety Science, vol. 47, no. 1, pp. 115-124, 2009.

[11] M.-H. Sigari, M. Fathy, and M. Soryani, "A driver face monitoring system for fatigue and distraction detection," International Journal of Vehicular Technology, vol. 2013, 2013.

[12] M. Patel, S. K. L. Lal, D. Kavanagh, and P. Rossiter, "Applying neural network analysis on heart rate variability data to assess driver fatigue," Expert Systems with Applications, vol. 38, no. 6, pp. 7235-7242, 2011.

[13] M. Zhou, X. Qu, and X. Li, "A recurrent neural network based microscopic car following model to predict traffic oscillation," Transportation Research Part C: Emerging Technologies, vol. 84, pp. 245-264, 2017.

[14] M. Zhou, X. Qu, and S. Jin, "On the Impact of Cooperative Autonomous Vehicles in Improving Freeway Merging: A Modified Intelligent Driver Model-Based Approach," IEEE Transactions on Intelligent Transportation Systems, vol. 18, no. 6, pp. 1422-1428, 2017.

[15] Z. Liu, Y. Zhang, S. Wang, and Z. Li, "A Trial-and-Error Method with Autonomous Vehicle-to-Infrastructure Traffic Counts for Cordon-Based Congestion Pricing," Journal of Advanced Transportation, vol. 2017, 2017.

[16] X. Qu, Y. Yang, Z. Liu, S. Jin, and J. Weng, "Potential crash risks of expressway on-ramps and off-ramps: a case study in Beijing, China," Safety Science, vol. 70, pp. 58-62, 2014.

[17] Q. Meng and J. Weng, "Evaluation of rear-end crash risk at work zone using work zone traffic data," Accident, Analysis \& Prevention, vol. 43, no. 4, pp. 1291-1300, 2011.

[18] M. Matowicki and O. Pribyl, "Speed compliance in freeway variable speed limit system -case study of the Prague city ring," Transport Problems, vol. 11, no. 1, pp. 69-78, 2016.

[19] X. Qu, J. Zhang, and S. Wang, "On the stochastic fundamental diagram for freeway traffic: Model development, analytical properties, validation, and extensive applications," Transportation Research Part B: Methodological, vol. 104, pp. 256-271, 2017.

[20] X. Qu, S. Wang, and J. Zhang, "On the fundamental diagram for freeway traffic: a novel calibration approach for single-regime models," Transportation Research Part B: Methodological, vol. 73, pp. 91-102, 2015.

[21] Z. Xu, T. Wei, S. Easa, X. Zhao, and X. Qu, "Modeling Relationship between Truck Fuel Consumption and Driving Behavior Using Data from Internet of Vehicles," Computer-Aided Civil and Infrastructure Engineering, vol. 33, no. 3, pp. 209-219, 2018.

[22] P. A. Hoggarth, C. R. H. Innes, J. C. Dalrymple-Alford, and R. D. Jones, "Prediction of driving ability: Are we building valid models?” Accident Analysis \& Prevention, vol. 77, pp. 29-34, 2015.
[23] K. A. Suhr and C. S. Dula, "The dangers of rumination on the road: Predictors of risky driving," Accident Analysis \& Prevention, vol. 99, pp. 153-160, 2017.

[24] W. Qi, H. Wen, Y. Wu, and L. Qin, "Effect model of urban traffic congestion on driver's lane-changing behavior," Advances in Mechanical Engineering, vol. 9, no. 9, pp. 1-12, 2017.

[25] A. P. Larocca, R. L. Ribeiro, A. da Cruz Figueira, P. T. de Oliveira, L. C. Lulio, and M. A. Rangel, "Analysis of perception of vertical signaling of highways by drivers in a simulated driving environment," Transportation Research Part F: Traffic Psychology and Behaviour, vol. 58, pp. 471-487, 2018.

[26] J. Dien, "Best practices for repeated measures ANOVAs of ERP data: Reference, regional channels, and robust ANOVAs," International Journal of Psychophysiology, vol. 111, pp. 42-56, 2017.

[27] B. M. Ozyildirim and M. Avci, "Generalized classifier neural network," Neural Networks, vol. 39, pp. 18-26, 2013.

[28] Ö. Polat and T. Yıldırım, "Genetic optimization of GRNN for pattern recognition without feature extraction," Expert Systems with Applications, vol. 34, no. 4, pp. 2444-2448, 2008. 


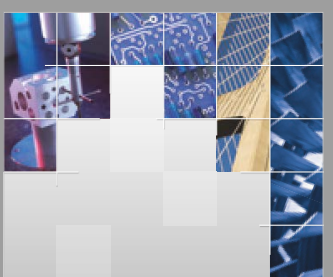

\section{Enfincering}
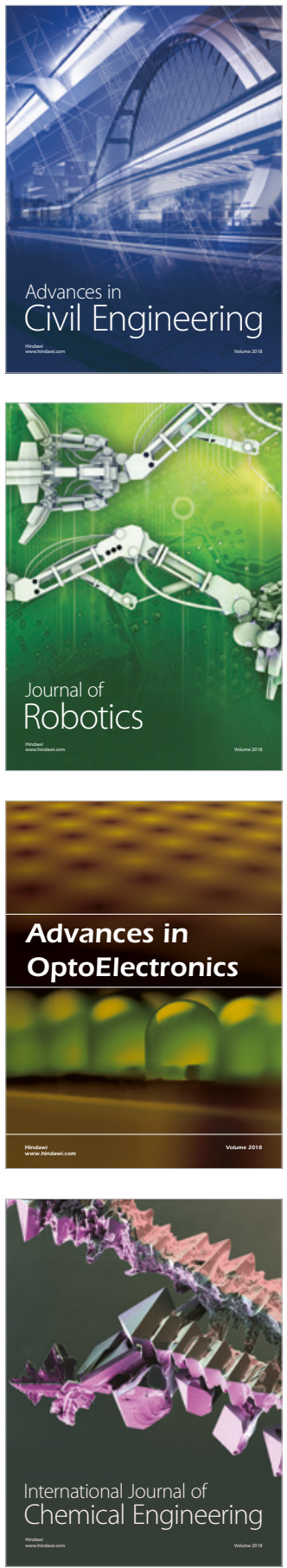

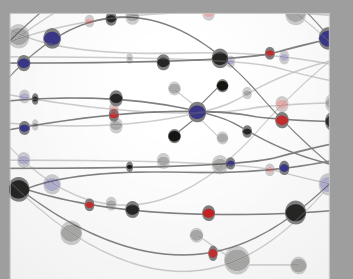

\section{Rotating \\ Machinery}

The Scientific World Journal

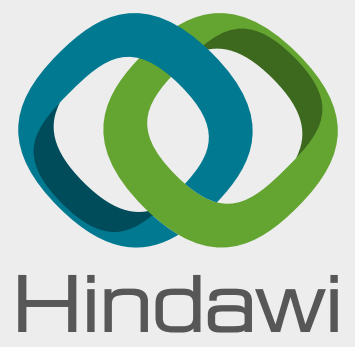

Submit your manuscripts at

www.hindawi.com
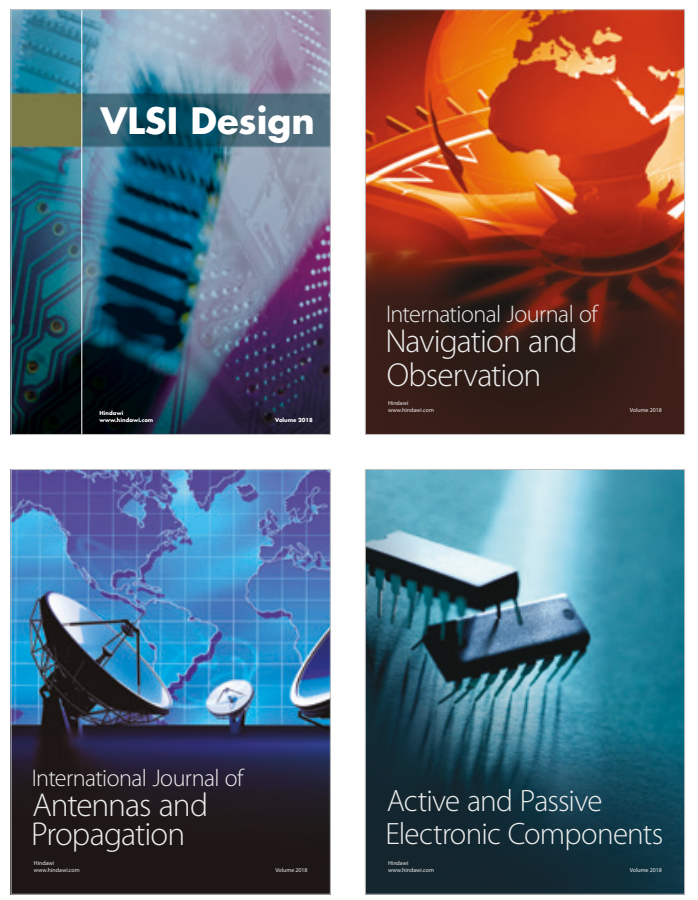
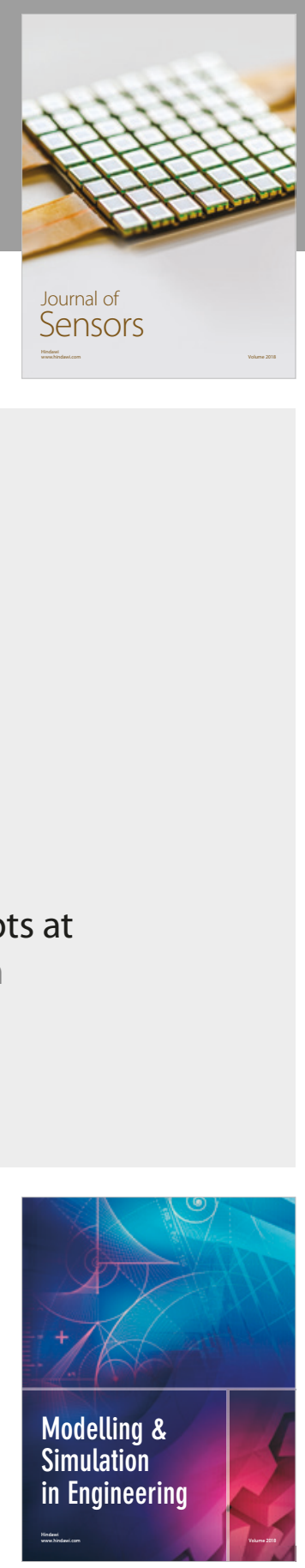

\section{Advances \\ Multimedia}
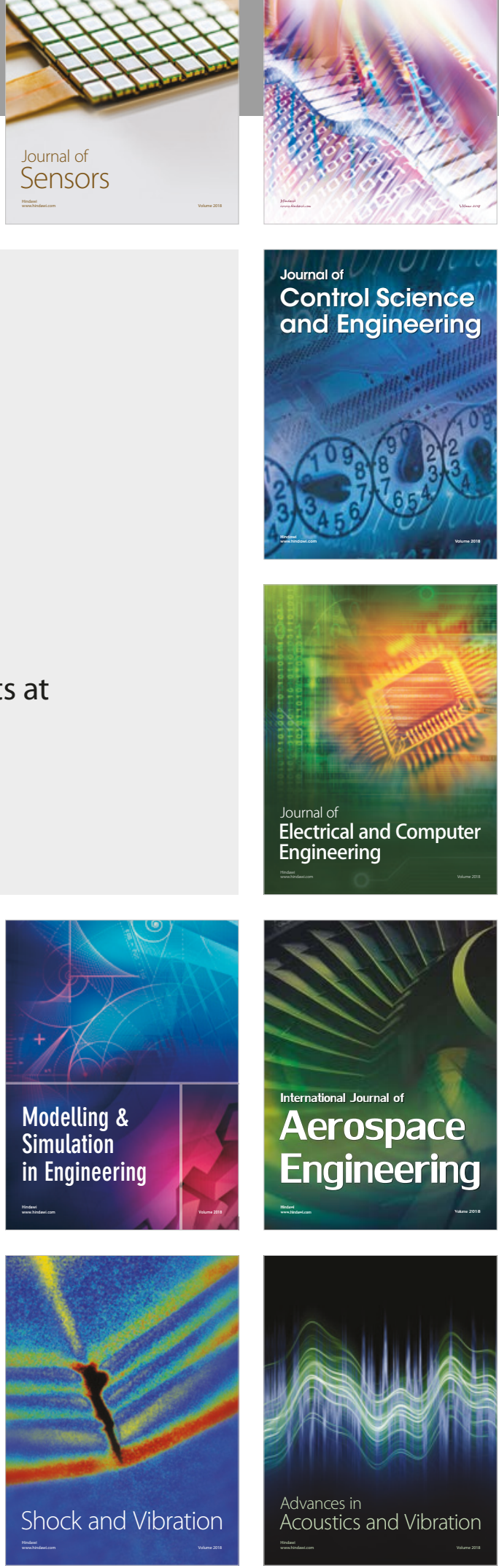